\title{
Structural phase transition and spontaneous interface reconstruction in $\mathrm{La}_{2 / 3} \mathrm{Ca}_{1 / 3} \mathrm{MnO}_{3} / \mathrm{BaTiO}_{3}$ superlattices
}

\author{
S. Turner, ${ }^{1, *}$ O. I. Lebedev, ${ }^{2}$ J. Verbeeck, ${ }^{1}$ K. Gehrke, ${ }^{3}$ V. Moshnyaga, ${ }^{3}$ and G. Van Tendeloo ${ }^{1}$ \\ ${ }^{1}$ EMAT, University of Antwerp, Groenenborgerlaan 171, 2020 Antwerp, Belgium \\ ${ }^{2}$ CRISMAT, UMR 6508, CNRS-ENSICAEN, 6 Bd Marechal Juin, 14050 Caen, France \\ ${ }^{3}$ Erstes Physikalisches Institut, Universität Göttingen, Friedrich-Hund-Platz 1, 37077 Göttingen, Germany
}

(Received 29 March 2012; revised manuscript received 18 December 2012; published 22 January 2013)

\begin{abstract}
$\left(\mathrm{La}_{2 / 3} \mathrm{Ca}_{1 / 3} \mathrm{MnO}_{3}\right)_{n} /\left(\mathrm{BaTiO}_{3}\right)_{m}\left(\mathrm{LCMO}_{n} / \mathrm{BTO}_{m}\right)$ superlattices on $\mathrm{MgO}$ and $\mathrm{SrTiO}_{3}$ substrates with different layer thicknesses $(n=10,38,40$ and $m=5,18,20)$ have been grown by metal organic aerosol deposition (MAD) and have been fully characterized down to the atomic scale to study the interface characteristics. Scanning transmission electron microscopy combined with spatially resolved electron energy-loss spectroscopy provides clear evidence for the existence of atomically sharp interfaces in MAD grown films, which exhibit epitaxial growth conditions, a uniform normal strain, and a fully oxidized state. Below a critical layer thickness the LCMO structure is found to change from the bulk Pnma symmetry to a pseudocubic $R \overline{3} c$ symmetry. An atomically flat interface reconstruction consisting of a single Ca-rich atomic layer is observed on the compressively strained BTO on LCMO interface, which is thought to partially neutralize the total charge from the alternating polar atomic layers in LCMO as well as relieving strain at the interface. No interface reconstruction is observed at the tensile strained LCMO on BTO interface.
\end{abstract}

DOI: 10.1103/PhysRevB.87.035418

PACS number(s): 68.35.-p, 68.37.Ma, 73.63.Bd

\section{INTRODUCTION}

With the discovery of a two-dimensional (2D) electron gas at the interface of two insulating oxide materials $\mathrm{LaAlO}_{3}$ (LAO) and $\mathrm{SrTiO}_{3}(\mathrm{STO})$, a renewed interest in the fine structure of interfaces in oxide based materials was sparked. ${ }^{1}$ This resulted in a large amount of research on different perovskitebased materials and various interfaces. ${ }^{2,3}$ In the LAO/STO system, properties such as magnetism and superconductivity have been observed while the coupling of different layers and tuning of the electron gas opened ways to explore these materials in prototype electronic devices. ${ }^{4,5}$ Within this class of complex oxides, manganites play a significant role. An example of such a compound is the $\mathrm{La}_{1-x} \mathrm{M}_{x} \mathrm{MnO}_{3}(\mathrm{M}=\mathrm{Sr}$, $\mathrm{Ca}, \mathrm{Ba}, \ldots$...) system which displays an intricate phase diagram depending on the doping level $x .{ }^{6}$ Between the insulating phase for both high and low $x$ and the ferromagnetic metallic phase for $x$ around 1/3, a broad region with electronic phase coexistence exists. ${ }^{7-9}$ In several compounds, e.g., $\mathrm{La}_{1-x} \mathrm{Ba}_{x} \mathrm{MnO}_{3}$ (LBMO) ${ }^{10}$ and $\mathrm{La}_{1-x} \mathrm{Ca}_{x} \mathrm{MnO}_{3}$ (LCMO), ${ }^{11}$ a large magnetic field dependency of the electrical resistance was measured, called colossal magnetoresistance (CMR) by Jin et al. ${ }^{11}$ These materials are of particular interest in spintronic applications such as spin filters due to their half-metallic state. ${ }^{12-15}$

Epitaxial multilayer structures or superlattices (SLs) based on manganites and other perovskite materials have been extensively studied in the light of enhancement or combination of the individual properties or multiferroic behavior. ${ }^{16-18}$ LCMO has been used in combination with $\mathrm{BaTiO}_{3}$ (BTO) which displays a magnetocapacitance effect ${ }^{19}$ and with $\mathrm{Pb}\left(\mathrm{Zr}_{1-x} \mathrm{Ti}_{x}\right) \mathrm{O}_{3}$ to form a field effect transistor. ${ }^{20}$ In order to expand these applications, a precise control of the growth process is crucial as the properties of thin films change dramatically depending on the layer structure and the detailed structure of the interfaces. ${ }^{21-23}$ Beside the stoichiometric and chemical composition, strain behavior also has to be taken into account as it plays an important role for the multiferroic properties. ${ }^{21,24-31}$ It was previously demonstrated that applying $2 \mathrm{D}$ or $3 \mathrm{D}$ stress to a LCMO film can change the structure of the LCMO from the bulk Pnma symmetry to a monoclinic $P 21 c^{32}$ or a pseudocubic $R \overline{3} c$ symmetry. ${ }^{33,34}$ In SLs the lattice mismatch induced strain can be tuned by varying the thickness $(n$ and $m$ ) of the layers.

Most of the structures mentioned above have been grown using pulsed laser deposition (PLD), a well-established technique where a high power laser ablates the target material and transfers it to the substrate in the form of a plasma plume. This procedure has been demonstrated to supply atomically flat, epitaxial oxide SLs. ${ }^{24}$ As the process has to be carried out under high vacuum conditions, great care has to be taken to avoid or control oxygen vacancies. ${ }^{25,26}$ An elegant, high oxygen pressure alternative to PLD growth of SLs is provided by metal organic aerosol deposition (MAD), which as a solutionbased and vacuum-free chemical deposition technique uses metal-chelate coordination compounds dissolved in an organic solvent (e.g., dimethylformamide) as precursors. ${ }^{35}$ SL samples are prepared by sequential spraying of controlled volumes of the corresponding precursor solutions onto a heated substrate at ambient oxygen pressure $\left(\mathrm{pO}_{2}=0.21 \mathrm{bar}\right)$.

In this work, superlattices of $\left[\mathrm{LCMO}_{n} / \mathrm{BTO}_{m}\right]_{10}$ with nominal thicknesses $n=10,38,40$ and $m=5,18,20$, respectively, are grown on $\mathrm{STO}(100)$ and $\mathrm{MgO}(100)$ substrates simultaneously by means of the MAD technique. The magnetotransport properties of the superlattices are presented in full, although some of the properties have already been published elsewhere. ${ }^{36}$ Advanced transmission electron microscopy addresses the structure and chemical composition of the SLs in detail by means of electron diffraction (ED), high resolution transmission electron microscopy (HRTEM), aberrationcorrected scanning transmission electron microscopy (STEM), and electron energy-loss spectroscopy (EELS). Our results show the existence of a critical layer thickness $d_{c} \cong 15 \mathrm{~nm}$ below which the LCMO structure changes from the bulk Pnma 
symmetry to a pseudocubic $R \overline{3} c$ symmetry, in agreement with earlier work in the literature. The former structure could be of interest for artificial multiferroic behavior as magnetic-field-induced switching of sample capacitance was observed for SLs with LSMO in Pnma symmetry. A spontaneous interface reconstruction is observed at the BTO on LCMO interface in SLs just below this critical thickness, namely in $\left[\mathrm{LCMO}_{38} / \mathrm{BTO}_{18}\right]_{10}$, grown on STO and MgO. The reconstruction consists of a single $\mathrm{Ca}$-rich atomic layer, which is thought to neutralize charge build up from alternating polar atomic planes in LCMO and relieve some of the strain present at the interface. No such interface reconstruction is found to be present at the tensile strained LCMO on BTO interface.

\section{EXPERIMENTAL SECTION}

MAD is a solution-based chemical deposition technique which uses metal-chelate coordination compounds as precursors, dissolved in an organic solvent (dimethylformamide). For the LCMO layers acetylacetonates of $\mathrm{La}, \mathrm{Mn}$ and $\mathrm{Ca}$ were employed, whereas the BTO layers were grown by using $\mathrm{Ba}$ (tmhd) $)_{2}$ and isoprop(Ti)(thmd) $)_{2}$ (thmd $=2,2,6,6$ tetramethylheptanedion). The sample preparation includes sequential deposition of LCMO and BTO layers at ambient oxygen pressure by means of spraying of controllable volumes of the corresponding LCMO and BTO solutions onto the heated substrate. The deposition was started with the LCMO layer and finished with the BTO one. The substrate temperature during deposition was kept at $T=960{ }^{\circ} \mathrm{C}$ and the growth time for individual LCMO and BTO layers was 30 and $16 \mathrm{~s}$, respectively. After deposition the samples were cooled down to room temperature in $30 \mathrm{~min}$.

The transport properties were measured in a four-probe geometry with ac current $I=100 \mu \mathrm{A} @ f=17 \mathrm{~Hz}$. In addition a coplanar capacitance, formed by two Au contacts at a distance of $20 \mu \mathrm{m}$ on the top BTO layer, was measured as a function of temperature and magnetic field using an ac bridge at $f=1 \mathrm{kHz}$.

The samples for ED and HRTEM investigation were prepared by ion milling using a Balzers ion mill. ED and HRTEM experiments were performed on a JEOL 4000EX microscope operated at $400 \mathrm{kV}$, with a point resolution of $1.7 \AA$. The samples for HAADF-STEM and EELS measurements were prepared by focused ion beam in a FEI Helios NanoLab 650 FIB. The HAADF-STEM images and EELS spectra were recorded on an aberration corrected FEI $\operatorname{Titan}^{3}$ microscope equipped with an aberration corrector for the probe-forming lens and a monochromator. The data in Figs. 4 and 5 were acquired at $300 \mathrm{kV}$, using a convergence semiangle $\alpha$ of $21.5 \mathrm{mrad}$ and an acceptance semiangle $\beta$ of $50 \mathrm{mrad}$ for imaging and 94 mrad for spectroscopy. The maps in Figs. 4, 5 , and 6 were generated by plotting the intensity under the background-subtracted EELS edges using an appropriate energy window. The data in Fig. 5(b) were processed by principle component noise filtering prior to mapping; no data treatment was applied to any of the other data sets. The monochromated EELS spectra in Fig. 6 were acquired on the same instrument operated at $120 \mathrm{kV}$ acceleration voltage, with the monochromator excited to provide an energy resolution of $250 \mathrm{meV}$ using a convergence semiangle $\alpha$ of $18 \mathrm{mrad}$ and an acceptance semiangle $\beta$ of $200 \mathrm{mrad}$ for spectroscopy and imaging.

\section{RESULTS AND DISCUSSION}

To gain insight into the local structure of the SLs, the chemical and structural nature of the films was investigated by advanced transmission electron microscopy. As the properties of perovskite materials are closely related to their structure, the latter was examined using electron diffraction and high resolution TEM. Figure 1 shows cross-sectional electron diffraction patterns and high resolution TEM images of the multilayer samples with different layer thicknesses $(n, m)$ : (a) $\mathrm{LCMO}_{10} / \mathrm{BTO}_{5}$ on $\mathrm{MgO}(100)$, (b) $\mathrm{LCMO}_{38} / \mathrm{BTO}_{18}$ on $\mathrm{STO}(100)$, and (c) $\mathrm{LCMO}_{40} / \mathrm{BTO}_{20}$ on $\mathrm{MgO}(100)$. All SLs have been grown
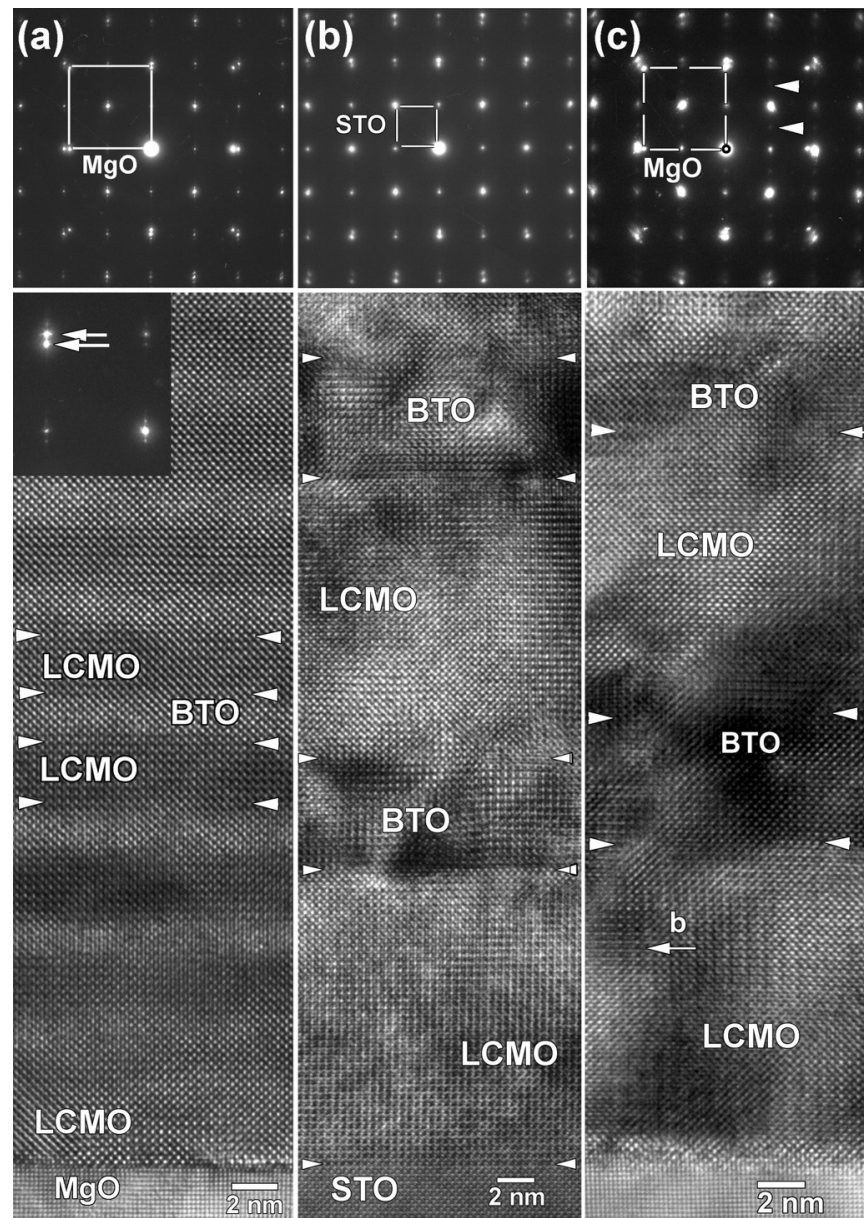

FIG. 1. Cross-sectional electron diffraction and high resolution TEM images of (a) a $\mathrm{LCMO}_{10} / \mathrm{BTO}_{5}$ multilayer grown on $\mathrm{MgO}(100)$, (b) a $\mathrm{LCMO}_{38} / \mathrm{BTO}_{18}$ multilayer grown on $\mathrm{STO}(100)$, and (c) a $\mathrm{LCMO}_{40} / \mathrm{BTO}_{20}$ multilayer grown on $\mathrm{MgO}(100)$, imaged along the [001] zone axis for $\mathrm{STO} / \mathrm{MgO}$. The arrowheads indicate the apparent layer boundaries. Below the $d_{c}\left(\mathrm{LCMO}_{10} / \mathrm{BTO}_{5}\right.$ and $\mathrm{LCMO}_{38} / \mathrm{BTO}_{18}$ ) the LCMO structure has pseudocubic $R \overline{3} c$ symmetry on both STO and $\mathrm{MgO}$. Above $d_{c}\left(\mathrm{LCMO}_{40} / \mathrm{BTO}_{20}\right)$ the LCMO structure has the bulk Pnma symmetry on both STO and $\mathrm{MgO}$, as evidenced by the extra reflections in the electron diffraction pattern. The epitaxial relationship is $(100)_{\mathrm{BTO}}\left\|(100)_{\mathrm{LCMO}}\right\|(100)_{\mathrm{STO} / \mathrm{MgO}}$ and $[001]_{\mathrm{BTO}}\left\|[001]_{\mathrm{LCMO}}\right\|[001]_{\mathrm{STO} / \mathrm{MgO}}$ in the pseudocubic notation. 
epitaxially and the epitaxial relationship, determined from HRTEM and ED data, is $(100)_{\mathrm{BTO}} / /(100)_{\mathrm{LCMO}} / /(100)_{\mathrm{STO} / \mathrm{MgO}}$ and $[001]_{\mathrm{BTO}} / /[001]_{\mathrm{LCMO}} / /[001]_{\mathrm{STO} / \mathrm{MgO}}$ in the pseudocubic notation for LCMO and BTO. It is at this point important to point out that the structural characteristics of the SLs grown on STO and $\mathrm{MgO}$ are almost identical, meaning that the properties of the various layers can be compared, even when they are grown on different substrates. A perfect epitaxial growth from the first LCMO layer on the substrate can be seen in Fig. 1. Atomically flat layers with predictable thicknesses $(n, m)$ are repeated over a total thickness of more than $200 \mathrm{~nm}$, evidencing a strong potential of MAD for synthesis of extended SL structures.

It is well known from literature that bulk LCMO exhibits an orthorhombically distorted perovskite-based structure of Pnma symmetry. ${ }^{6}$ However, based upon the ED results, the following space groups can be assigned to the observed layers in the case of $\mathrm{LCMO}_{10} / \mathrm{BTO}_{5}$ and $\mathrm{LCMO}_{38} / \mathrm{BTO}_{18}$ [Figs. 1(a) and 1(b)]: Pseudocubic $R \overline{3} c$ for LCMO and a tetragonally distorted perovskite unit cell with symmetry $P 4 / \mathrm{mm}$ for BTO. In other words, for all SLs below a critical LCMO thickness, LCMO undergoes a phase transition to a pseudocubic $R \overline{3} c$ structure. Under relaxed conditions, i.e., in bulk, the pseudocubic lattice parameters are $3.905 \AA$ for STO, $4.018 \AA$ for BTO, and $3.87 \AA$ for LCMO. Therefore, LCMO, if grown on BTO, would suffer a tensile strain, whereas BTO on LCMO would be compressively strained. In the case of $\mathrm{LCMO}_{40} / \mathrm{BTO}_{20}$ [Fig. 1(c)], which is a SL with only a slightly increased layer thickness with respect to $\mathrm{LCMO}_{38} / \mathrm{BTO}_{18}$, the LCMO relaxes to the bulk Pnma structure on both STO and $\mathrm{MgO}$ substrates, as evidenced by the extra reflections at $7.8 \AA$ in the electron diffraction pattern in Fig. 1(c). This is confirmed by the corresponding HRTEM image where typical contrast for the orthorhombic LCMO structure is observed. This means that the critical layer thickness $d_{c}$ for LCMO must lie just above 38 unit cells (u.c.), i.e., approximately $15 \mathrm{~nm}$. The reconstruction to the relaxed Pnma structure relieves the elastic energy buildup, which is dependent upon the film thickness $d$ and the lattice constant of the two crystal structures in the SL, above the critical layer thickness.

Although within MAD growth conditions $\left(p \mathrm{O}_{2}=0.21 \mathrm{bar}\right)$ the formation of oxygen vacancies should be suppressed, we performed extra measurements to exclude charge effects, i.e., oxygen vacancies, as the driving force of the altered space group. As a proof of full oxygenation of the MAD grown perovskite films thick $\mathrm{LaMnO}_{3}$ (LMO) layers, grown under similar conditions, did indeed display the fully oxidized $R \overline{3} c$ structure (see Supplementary Material ${ }^{49}$ ). Together with the altered point group of LCMO, strain maps, obtained by a geometric phase analysis (GPA), ${ }^{37}$ unambiguously show coherently strained LCMO layers in the case of $\mathrm{LCMO}_{10} / \mathrm{BTO}_{5}$ and $\mathrm{LCMO}_{38} / \mathrm{BTO}_{18}$ (Fig. 2). The GPA patterns point out a uniform strain in the direction normal to the growth direction $\left(g_{020}\right)$, consistent with different pseudocubic lattice parameters of the constituents, and almost no in-plane strain as supplementary evidence for the excellent growth performance $\left(g_{200}\right)$. When $\mathrm{MgO}$ is used as substrate, the lattice mismatch between $\mathrm{MgO}$ and LCMO is accommodated by inserting misfit dislocations approximately every $3 \mathrm{~nm}$.

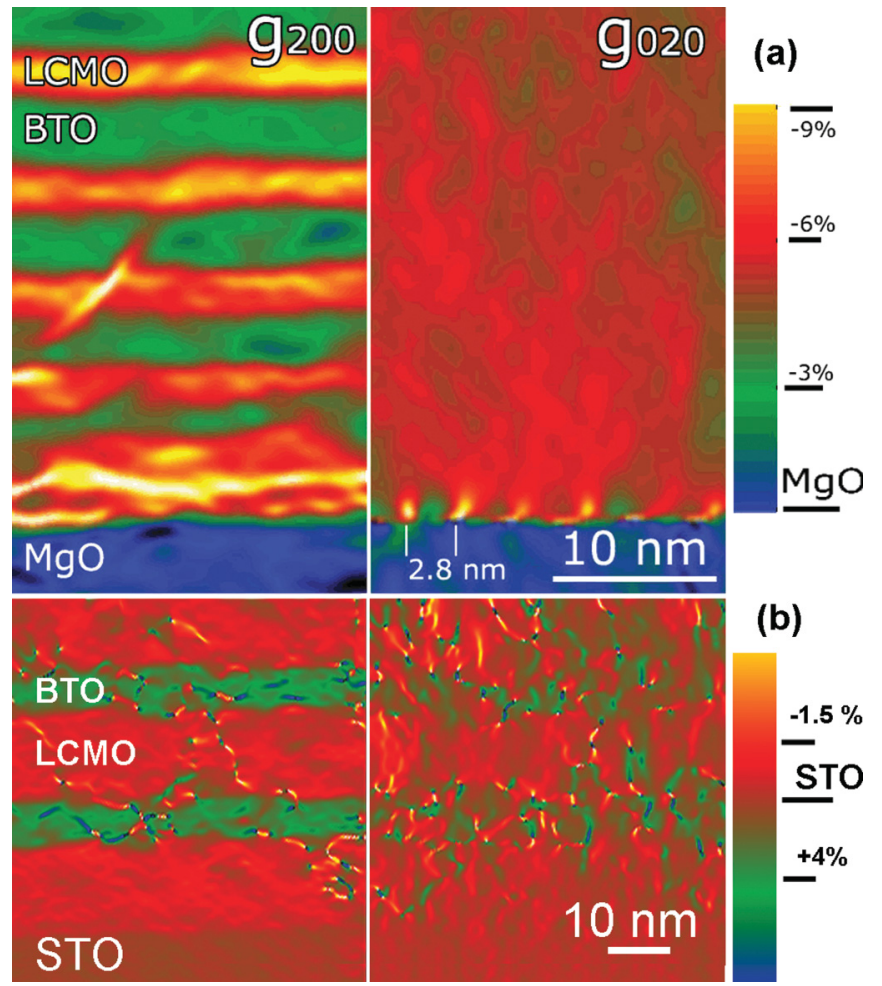

FIG. 2. (Color online) HRTEM GPA strain analysis maps of the strain component parallel to the interface $\left(g_{200}\right)$ and perpendicular to the interface $\left(g_{020}\right)$ for (a) $\mathrm{LCMO}_{10} / \mathrm{BTO}_{5}$ grown on $\mathrm{MgO}(100)$ and (b) $\mathrm{LCMO}_{38} / \mathrm{BTO}_{18}$ grown on $\mathrm{STO}(100)$. In the case of $\mathrm{MgO}$ periodical misfit dislocations are present to accommodate the lattice mismatch. $\mathrm{LCMO}_{38} / \mathrm{BTO}_{18}$ on $\mathrm{STO}(100)$, with $d_{\mathrm{LCMO}}<d_{c}$, still shows coherently strained LCMO and BTO layers with low residual in-plane strain due to lattice misfit. In both SLs the LCMO has an altered pseudocubic $R \overline{3} c$ structure.

The transport properties of SLs with LCMO layer thickness $n=38$ (grown on STO) and $n=40$ (grown on $\mathrm{MgO}$ ), i.e., within $R \overline{3} c$ and Pnma structure, respectively, were measured in a four-probe geometry and are presented in Fig. 3. The STO sample shows a very low metal-insulator transition temperature in ambient magnetic field $T_{\mathrm{MI}} \sim 100-130 \mathrm{~K}$ (estimated), as well as very large CMR $=100 \% *[R(0 \mathrm{~T})-$ $R(7 \mathrm{~T})] / R(7 \mathrm{~T}) \sim 10^{5} \%$. In contrast, the $\mathrm{MgO}$ sample reveals a significantly larger $T_{\mathrm{MI}}=220 \mathrm{~K}$ and a moderate $\mathrm{CMR} \sim 900 \%$ [see Fig. 3(b)]. These results are in line with the above structural analysis (Fig. 2), which evidences a coherently strained $\mathrm{LCMO}_{38}$ layer with $R \overline{3} c$ structure in the SL grown on STO and relaxed $\mathrm{LCMO}_{40}$ with Pnma structure in the SL grown on $\mathrm{MgO}$. The capacitance as a function of temperature for the STO sample [see the inset to Fig. 3(a)] shows a huge CMR-induced minimum ${ }^{38}$ for $T=130 \mathrm{~K}$, which is very close to $T_{\mathrm{MI}} @ B=0$. In contrast, the capacitance for the $\mathrm{MgO}$ sample [see the inset to Fig. 3(b)] is almost temperature independent for $T<200 \mathrm{~K}$. Note that the value of the geometrical capacitance for both samples is very similar, $C \sim 10-14 \mathrm{nF}$. A remarkable difference between these two samples is the observed switching of the capacitance under applied magnetic field in the $\mathrm{MgO}$ sample [Fig. 3(c)]. The switching is hysteretic and occurs in the temperature range 

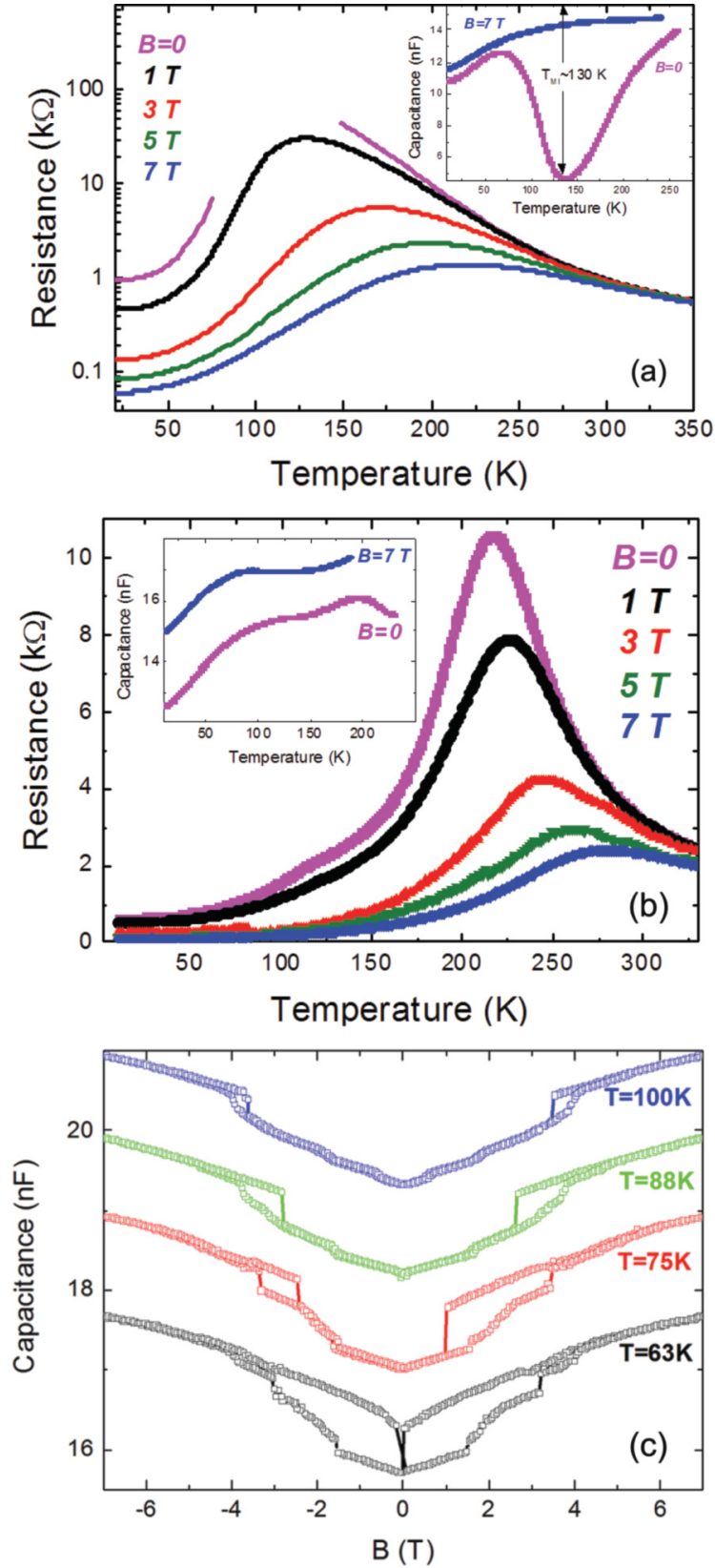

FIG. 3. (Color online) Temperature dependencies of the resistance for the (a) $\mathrm{LCMO}_{38} / \mathrm{BTO}_{18} \mathrm{SL}$ grown on STO, and for (b) $\mathrm{LCMO}_{40} / \mathrm{BTO}_{20}$ on $\mathrm{MgO}$. The insets demonstrate the corresponding temperature behavior of the capacitance. (c) Hysteretic switching of the capacitance in applied magnetic field for the $\mathrm{LCMO}_{40} / \mathrm{BTO}_{20}$ $\mathrm{SL}$ grown on $\mathrm{MgO}$. The curves for $T=63,75,88$, and $100 \mathrm{~K}$ are shifted by $2 \mathrm{nF}$ for clarity.

$T=50-150 \mathrm{~K}$, where the CMR effect is suppressed. Taking into account that the large "geometric" capacitance originates from the insulating BTO, we interpret the capacitance switching as a reversal of the ferroelectric domains by a magnetic field due to a strong magneto electric and possibly elastic coupling between LCMO and BTO layers. These results hint towards multiferroic behavior in the $\mathrm{MgO}$ sample with strain relaxed $\mathrm{LCMO}_{40}$ (Pnma) and $\mathrm{BTO}_{20}$ layers, which are not clamped with each other and, thus, allow an elastic coupling.
As we observed and discussed above, the thickness of the LCMO layer in the coherently strained $\mathrm{LCMO}_{38} / \mathrm{BTO}_{18}$ SL lies just below the critical layer thickness. This makes the interfaces in this SL particularly interesting for a more detailed investigation. As the strain and charge buildup in the LCMO layers of this SL must be substantial, a compensation mechanism might be present. In fact, comparing the interfaces in $\mathrm{LCMO}_{10} / \mathrm{BTO}_{5}$ and $\mathrm{LCMO}_{38} / \mathrm{BTO}_{18}$ in the HRTEM images in Fig. 1, the sharp BTO on LCMO interface in $\mathrm{LCMO}_{38} / \mathrm{BTO}_{18}$ is striking, when compared to the apparently more diffuse LCMO on BTO interface and the interfaces in $\mathrm{LCMO}_{10} / \mathrm{BTO}_{5}$. To investigate the interface structure in the case of $\mathrm{LCMO}_{38} / \mathrm{BTO}_{18}$, detailed high-angle annular dark field STEM (HAADF-STEM) and electron energy-loss spectroscopy experiments were carried out. As the structure of the SLs is similar on both STO and MgO but the misfit is smaller in the case of STO, all further results presented were obtained on $\mathrm{LCMO}_{38} / \mathrm{BTO}_{18}$ grown on an STO substrate. A typical HAADF-STEM image of the multilayer structure on STO is depicted in Fig. 4(a) where the STO substrate layer shows up darker because of the lower average atomic number. Although the average density of LCMO and BTO is quite similar, the different layers are distinguished as intensity variations with sharp interfaces. Low magnification elemental maps were obtained simultaneously from
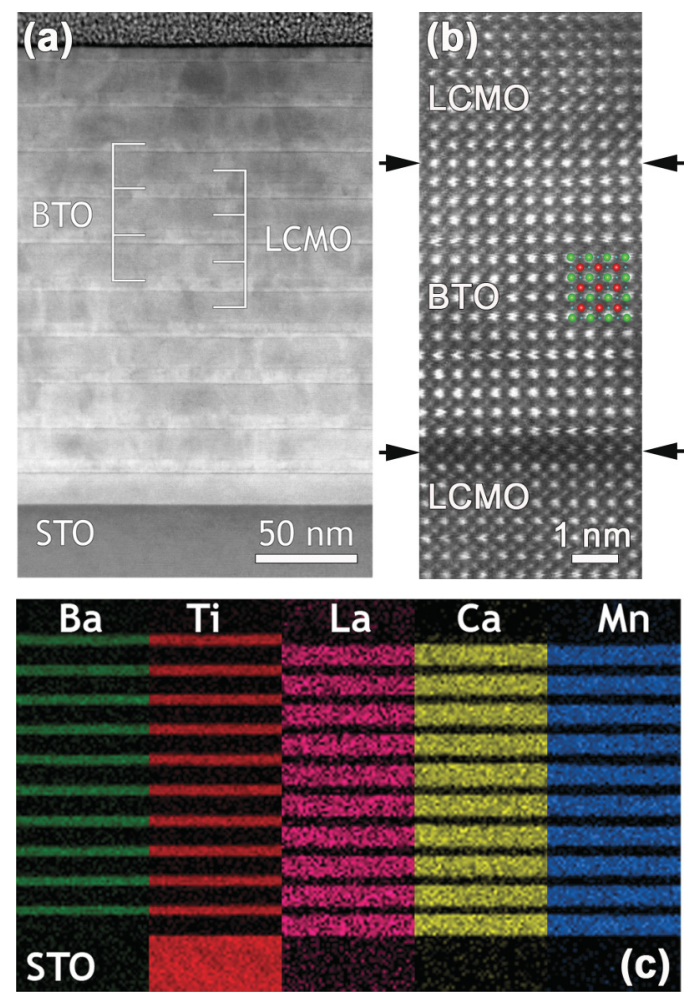

FIG. 4. (Color online) (a) Low magnification HAADF-STEM image of the $\mathrm{LCMO}_{38} / \mathrm{BTO}_{18}$ SL on STO. Alternating LCMO and BTO layers can be clearly distinguished. (b) High resolution HAADF image of both interfaces. Both A (green dots) and B cations (red dots) of the perovskite structures are resolved. The LCMO on BTO interface is relatively sharp (arrows), an atomically flat dark band of one unit layer is observed at the BTO on LCMO interface (arrows). (c) Overview STEM-EELS elemental maps of the heterostructure. 
STEM-EELS measurements by standard background subtraction and peak integration of the $\mathrm{Ba} M_{4,5}$, Ti $L_{2,3}$, La $M_{4,5}$, $\mathrm{Ca} L_{2,3}$, and $\mathrm{Mn} L_{2,3}$ edges. The result in Fig. 4(c) shows clear elemental separation as no diffusion at this length scale is observed between different layers. The higher noise level in the lanthanum map can be explained by the preceding Ba peak which forces the use of a narrow energy selecting window for background estimation and thus yields a noisier result. Even though the maps correspond well to the SL stacking sequence, these observations do not rule out a possible interdiffusion at the (sub) nanometer scale. This data does strongly confirm that all structure transformations are due to applied stress rather than chemical composition changes. On top of each LCMO layer, at the BTO on LCMO interface, a dark band is present in Fig. 4(a) that is even more striking in the magnified image [Fig. 4(b)]. The latter image suggests the insertion of a single layer with a lower average atomic number evidenced by its reduced intensity. This is repeated systematically at every interface and is perfectly flat, ruling out imaging artifacts or a thickness effects as the cause. No such dark band is visible at the LCMO on BTO interface, which also appears to be slightly less sharp as judged from the HAADF-STEM image intensity.

Figure 5(a) shows high resolution elemental maps of the five most important elements of this sample, Ba (green), Ti (red), $\mathrm{La}$ (pink), $\mathrm{Ca}$ (yellow), and Mn (blue) for the compressively strained BTO on LCMO interface, displaying the dark feature (indicated by a white arrow). It is immediately apparent from the maps that there is a relative offset of 1 monolayer between the $\mathrm{Ca}$ and the $\mathrm{La}$ map, indicating a higher $\mathrm{Ca} / \mathrm{La}$ ratio near the compressively strained BTO on LCMO interface (arrows). This explains the lower intensity of the atomic layer beneath the interface observed in the HAADF-STEM images: The lower atomic weight of $\mathrm{Ca}$ with respect to $\mathrm{La}$ yields a low-contrast band at the interface in the HAADF-STEM images. Figure 5(b) shows high resolution elemental maps for the tensile strained LCMO on BTO interface. In this case no dark band is visible in the HAADF-STEM image, and

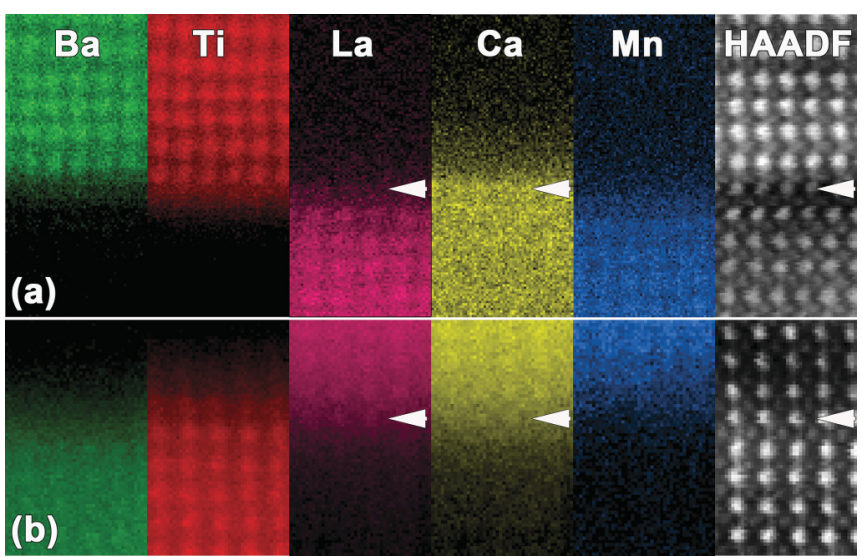

FIG. 5. (Color online) High resolution EELS elemental maps of barium, titanium, lanthanum, calcium, and manganese for the (a) compressively strained BTO on LCMO interface and (b) tensile strained LCMO on BTO interface. The elemental maps show a clear offset between the $\mathrm{La}$ and $\mathrm{Ca}$ lattice and single layer of Ca enrichment at the compressively strained interface (indicated by arrows), while no offset is present in for the tensile strained interface (arrows). the interface appears to be less sharp than the compressed interface. The atomic resolution elemental maps show that no offset between the $\mathrm{Ca}$ and $\mathrm{La}$ termination is present at this interface; both $\mathrm{Ca}$ and $\mathrm{La}$ terminate at the same atomic plane. Some diffusion of Ti into the Mn sublattice can however be made out from the atomic resolution elemental maps, stretching over approximately 1 unit cell. In other words, no A-cation diffusion or segregation is present at this interface, but a limited diffusion of $\mathrm{B}$ cations is often present (the Ti interdiffusion was not measured in all acquired EELS data sets).

Valency changes in the B-cation sublattice near to or at interfaces are known to occur in oxide SLs, and can be of vital importance in accommodating an electronic interface reconstruction. To investigate whether any changes in valency occur in the $\mathrm{Ti}$ or $\mathrm{Mn}$ sublattice of the $\mathrm{LCMO}_{38} / \mathrm{BTO}_{18} \mathrm{SL}$, the fine structure of the $\mathrm{Ti}$ and $\mathrm{Mn} L_{2,3}$ EELS edges was investigated using high resolution EELS. Both the shape and onset of these $\mathrm{Ti}$ and $\mathrm{Mn}$ edges are known to be closely related to their valence state. ${ }^{39,40}$ In Fig. 6 EELS data obtained with a monochromatic electron probe $(\Delta E=250 \mathrm{meV})$ from both the compressively strained BTO on LCMO and the tensile strained LCMO on BTO interface are plotted. No measurable changes in the fine structure were found over either of the interfaces; neither for the compressively strained BTO on LCMO interface, nor for the tensile strained LCMO on BTO interface. Thus, a significant change in the cation fine structure of the Ti $L_{2,3}$ or the Mn $L_{2,3}$ cannot be made out, indicating that an electronic reconstruction does not occur here.

Four main conclusions can be drawn from the atomic resolution EELS data. (1) There is a symmetric $\mathrm{TiO}_{2}$ plane ending of the BTO layers, which agrees with the recent observations of similar oxide heterostructures. ${ }^{40,41}$ (2) There is a clear asymmetric ( $\mathrm{LaCa}) \mathrm{O}$ termination of the LCMO layers. The termination of lanthanum and calcium coincide nicely on the LCMO on BTO interface which exhibits tensile strain in Fig. 5. In contrast, an offset of about $4 \AA$, i.e., one perovskite unit cell, is clearly visible on the BTO on LCMO interface with compressive strain. This gives strong support to the hypothesis of a single atomic layer with considerably higher calcium content at this interface and is consistent with measurements by Simon et al., where calcium segregation was found below a strained STO layer embedded in LCMO. ${ }^{42}$ (3) A limited diffusion of $\mathrm{Ti}$ cations into the Mn B-cation sublattice over approximately 1 unit cell is often present at the tensile strained LCMO on BTO interface. (4) No valency changes for $\mathrm{Ti}$ or $\mathrm{Mn}$ are measured over either of the interfaces. From the elemental maps we can establish a schematic stacking sequence; the stoichiometric compositions predict the alternation of charged $\left(\mathrm{La}_{2 / 3}^{3+} \mathrm{Ca}_{1 / 3}^{2+} \mathrm{O}^{2-}\right)^{+2 / 3}$. $\left(\mathrm{Mn}^{3.33+} \mathrm{O}_{2}^{2}\right)^{-2 / 3}$ layers for LCMO and neutral $\left(\mathrm{Ba}^{2+} \mathrm{O}^{2-}\right)^{0}-$ $\left(\mathrm{Ti}^{4+} \mathrm{O}_{2}^{2-}\right)^{0}$ layers for BTO. From our EELS data we find a nominal $\left(\mathrm{La}_{2 / 3} \mathrm{Ca}_{1 / 3} \mathrm{O}\right)-\left(\mathrm{MnO}_{2}\right)-(\mathrm{CaO})-\left(\mathrm{TiO}_{2}\right)-(\mathrm{BaO})-$ $\left(\mathrm{TiO}_{2}\right)-\cdots$ stacking for the compressive-strained interface and a $(\mathrm{BaO})-\left(\mathrm{TiO}_{2}\right)-\left(\mathrm{La}_{2 / 3} \mathrm{Ca}_{1 / 3} \mathrm{O}\right)-\left(\mathrm{MnO}_{2}\right)-\left(\mathrm{La}_{2 / 3} \mathrm{Ca}_{1 / 3} \mathrm{O}\right)-\cdots$ for the tensile-strained interface. This stacking is displayed schematically in Fig. 7.

The origin of the Ca segregation is most likely twofold: (1) The BTO on LCMO interface is compressively strained and 

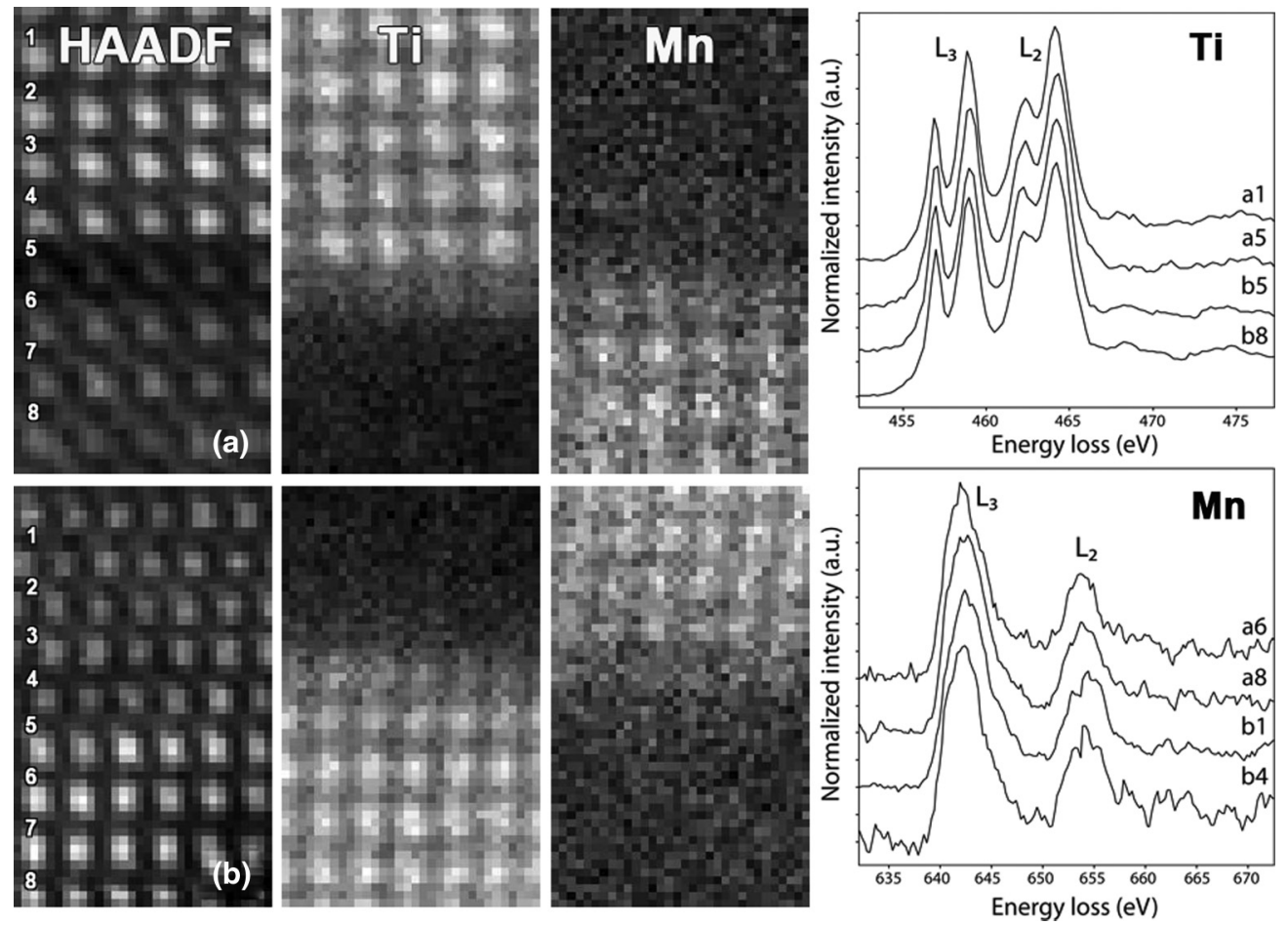

FIG. 6. Monochromated EELS spectroscopy of the (a) BTO on LCMO interface with the Ca-rich layer between planes $a 5$ and $a 6$ and the (b) LCMO on BTO interface. The Ti and Mn maps confirm the sharp interfaces in the material. Line averaged Ti $L_{2,3}$ and Mn $L_{2,3}$ EELS spectra ( 3 pixels width) from representative B cation planes at the interface and far from the interface. No fine structure changes that could indicate $\mathrm{a} \mathrm{Ti}(4+)$ or $\mathrm{Mn}(3.33+)$ valency change are apparent at the interface.

the layer thickness of the LCMO layer in the $\mathrm{LCMO}_{38} / \mathrm{BTO}_{18}$ is very close to the $d_{c}$, upon which LCMO switches to the bulk Pnma symmetry. Segregation of a single layer of $\mathrm{CaO}$, the atomic radius of $\mathrm{Ca}$ is $180 \mathrm{pm}$ while the atomic radius of $\mathrm{La}$ is $195 \mathrm{pm}$, should then alleviate some of the compressive stain built up at this interface when the LCMO layer is grown close to the critical layer thickness. (2) As both interfaces originate

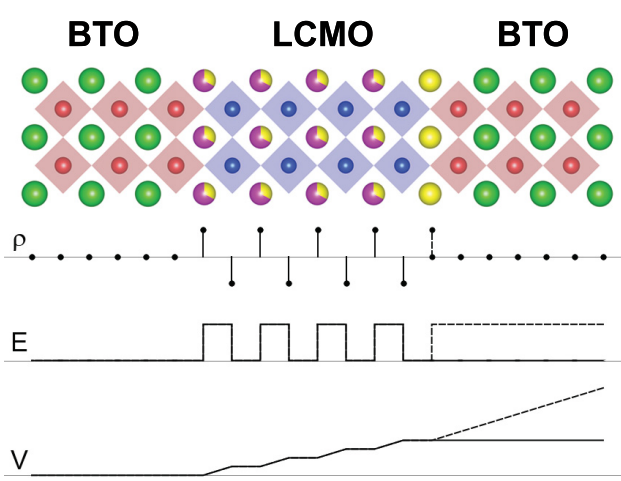

FIG. 7. (Color online) Helping to reduce the polar catastrophe by interface reconstruction. The top illustration shows a schematic stacking sequence of the observed multilayer system, where the same colors as before have been used (for visibility, the number of stacking layers has been reduced). The graphs show respectively the charge $(\rho)$, generated electric field $(E)$, and electrostatic potential $(V)$ for the reconstructed (solid line) and nonreconstructed (dashed line) interface at the position of every layer. The ending $\mathrm{CaO}$ layer renders the total LCMO layer electrically neutral and therefore reduces the electrostatic potential. from similar polar/nonpolar layers, it is also worthwhile to look at the effect of the altered interface on the so-called polar catastrophe, which gives rise to a divergence of the electrostatic potential. ${ }^{43}$ If we neglect any change in stoichiometry at the interface and rule out valence changes, the potential diverges linearly. The observed change in layer ending not only makes the whole LCMO layer electrically neutral (see Fig. 7) but also removes the polar divergence making the potential constant in the BTO layer. The charge neutrality always has been used as a strong argument in favor of a block-by-block growth mechanism of oxides. ${ }^{44,45}$ We indeed find a symmetric stacking sequence with an additional compositional change of the ending layer (partially) solving the charge problem and polarity mismatch. The polar catastrophe was originally explained by the charge transfer and accompanying valence changes of transition metal $\mathrm{B}$ cations of a perovskite. ${ }^{43}$ However, as shown by the high resolution EELS data above, no measurable changes in the fine structure were found over either of the interfaces, indicating that an electronic reconstruction does not occur here. This is similar to what was observed before by others in, e.g., the LAO/STO SLs. ${ }^{46}$ Thus, a compositional surface reconstruction rather than charge transfer seems to be a more realistic interpretation. A similar doping mechanism has already been introduced to alleviate the polar catastrophe in the case of $\mathrm{LAO} / \mathrm{STO} .^{47,48}$

\section{CONCLUSION}

$\mathrm{La}_{2 / 3} \mathrm{Ca}_{1 / 3} \mathrm{MnO}_{3} / \mathrm{BaTiO}_{3}$ superlattices have been grown on $\mathrm{MgO}$ and $\mathrm{SrTiO}_{3}$ substrates by metal organic aerosol 
deposition and fully characterized down to the atomic scale to study the crystal structure and interface characteristics. Scanning transmission electron microscopy provided clear evidence that MAD as a vacuum-free and solution-based chemical technique supplies high quality SLs in a controlled way, with atomically sharp interfaces. The films are epitaxially grown in a fully oxidized state with LCMO layers up to a critical thickness $d_{c} \sim 39$ u.c., exhibiting a uniform normal strain. Upon exceeding the critical LCMO layer thickness, a structural transformation ( $R-3 c$ to Pnma) occurs, leading to strain relaxation. A switching of the capacitance by magnetic field was detected in a SL with 40 u.c. thick LCMO layers with Pnma structure. Just below $d_{c}$, in $\mathrm{LCMO}_{38} / \mathrm{BTO}_{18}$, a chemically stabilized interface reconstruction was found to be present. Here an interface reconstruction consisting of a single $\mathrm{Ca}$-rich atomic layer was observed on the compressively strained BTO on LCMO interface, which is thought to stabilize charge build up from alternating nonpolar atomic planes as well as to relieve strain buildup. No interface reconstruction was found to be present at the tensile strained LCMO on BTO interface.

\section{ACKNOWLEDGMENTS}

S.T. acknowledges financial support from the Fund for Scientific Research-Flanders (FWO). This work was funded by the European Union Council under the 7th Framework Program (FP7) Grant No. 246102 IFOX. J.V. and G.V.T. acknowledge funding from the FP7, ERC Grant No. 246791 COUNTATOMS. The Titan microscope used for this study was partially funded by the HERCULES foundation of the Flemish Government. *stuart.turner@ua.ac.be

${ }^{1}$ A. Ohtomo and H. Y. Hwang, Nature (London) 427, 423 (2004).

${ }^{2}$ N. Reyren, S. Thiel, A. D. Caviglia, L. F. Kourkoutis, G. Hammerl, C. Richter, C. W. Schneider, T. Kopp, A.-S. Rüetschi, D. Jaccard, M. Gabay, D. A. Muller, J.-M. Triscone, and J. Mannhart, Science 317, 1196 (2007).

${ }^{3}$ A. Brinkman, M. Huijben, M. Van Zalk, J. Huijben, U. Zeitler, J. C. Maan, W. G. van der Wiel, G. Rijnders, D. H. A. Blank, and H. Hilgenkamp, Nat. Mater. 6, 493 (2007).

${ }^{4}$ S. Thiel, G. Hammerl, A. Schmehl, C. W. Schneider, and J. Mannhart, Science 313, 1942 (2006).

${ }^{5}$ M. Huijben, G. Rijnders, D. H. A. Blank, S. Bals, S. Van Aert, J. Verbeeck, G. Van Tendeloo, A. Brinkman, and H. Hilgenkamp, Nat. Mater. 5, 556 (2006).

${ }^{6}$ P. G. Radaelli, M. Marezio, H. Y. Hwang, S.-W. Cheong, and B. Batlogg, Phys. Rev. B 54, 8992 (1996).

${ }^{7}$ A.-M. Haghiri-Gosnet and J.-P. Renard, J. Phys. D: Appl. Phys. 36, R127 (2003).

${ }^{8}$ A. P. Ramirez, J. Phys.: Condens. Matter 9, 8171 (1997).

${ }^{9}$ E. Dagotto, T. Hotta, and A. Moreo, Phys. Rep. 344, 1 (2001).

${ }^{10}$ R. von Helmolt, J. Wecker, B. Holzapfel, L. Schultz, and K. Samwer, Phys. Rev. Lett. 71, 2331 (1993).

${ }^{11}$ S. Jin, T. H. Tiefel, M. McCormack, R. A. Fastnacht, R. Ramesh, and L. H. Chen, Science 264, 413 (1994).

${ }^{12}$ J. F. Gregg, I. Petej, E. Jouguelet, and C. J. Dennis, Phys. D: Appl. Phys. 35, R121 (2002).

${ }^{13}$ S. A. Wolf, D. D. Awschalom, R. A. Buhrman, J. M. Daughton, S. von Molnár, M. L. Roukes, A. Y. Chtchelkanova, and D. M. Treger, Science 294, 1488 (2001).

${ }^{14}$ J. S. Moodera, L. R. Kinder, T. M. Wong, and R. Meservey, Phys. Rev. Lett. 74, 3273 (1995).

${ }^{15}$ E. Y. Tsymbal and H. Kohlstedt, Science 313, 181 (2006).

${ }^{16}$ M. Gajek, M. Bibes, S. Fusil, K. Bouzehouane, J. Fontcuberta, A. Barthélémy, and A. Fert, Nat. Mater. 6, 296 (2007).

${ }^{17}$ K. Dorr, T. Walter, M. Sahana, K. Muller, K. Nenkov, K. Brand, and L. Schultz, J. Appl. Phys. 89, 6973 (2001).

${ }^{18}$ O. I. Lebedev, J. Verbeeck, G. Van Tendeloo, C. Dubordieu, M. Rosina, and P. Chaudouet, J. Appl. Phys. 94, 7646 (2003).

${ }^{19}$ M. P. Singh, W. Prellier, C. Simon, and B. Raveau, Appl. Phys. Lett. 87, 022505 (2005).
${ }^{20}$ S. Mathews, R. Ramesh, T. Venkatesan, and J. Benedetto, Science 276, 238 (1997).

${ }^{21}$ Z. Q. Yang, R. Hendrikx, J. Aarts, Y. L. Qin, and H. W. Zandbergen, Phys. Rev. B 70, 174111 (2004).

${ }^{22}$ Y. Ishii, H. Yamada, H. Sato, H. Akoh, Y. Ogawa, M. Kawasaki, and Y. Tokura, Appl. Phys. Lett. 89, 042509 (2006).

${ }^{23}$ H. Yamada, Y. Ogawa, Y. Ishii, H. Sato, M. Kawasaki, H. Akoh, and Y. Tokura, Science 305, 646 (2004).

${ }^{24}$ A. Ohtomo, D. A. Muller, J. L. Grazul, and H. Y. Hwang, Nature (London) 419, 378 (2002).

${ }^{25}$ A. Kalabukhov, R. Gunnarsson, J. Börjesson, E. Olsson, T. Claeson, and D. Winkler, Phys. Rev. B 75, 121404 (2007).

${ }^{26}$ Y. Davila, A. Petitmangin, C. Hebert, J. Perrière, and W. Seiler, Appl. Surf. Sci. 257, 5354 (2011).

${ }^{27}$ M. Ziese, H. C. Semmelhack, and H. H. Han, Phys. Rev. B 68, 134444 (2003)

${ }^{28}$ J. Cao and J. Wu, Mater. Sci. Eng. R. 71, 35 (2011).

${ }^{29}$ M. Paranjape, A. K. Raychaudhuri, N. D. Mathur, and M. G. Blamire, Phys. Rev. B 67, 214415 (2003).

${ }^{30}$ B. Vengalis, A. Maneikis, F. Anisimovas, R. Butkute, L. Dapkus, and A. Kindurys, J. Magn. Magn. Mater. 211, 35 (2000).

${ }^{31}$ N. A. Spaldin and M. Fiebig, Science 309, 391 (2005).

${ }^{32}$ O. I. Lebedev, G. Van Tendeloo, S. Amelinckx, B. Leibold, and H.-U. Habermeier, Phys. Rev. B 58, 8065 (1998).

${ }^{33}$ V. Moshnyaga, B. Damaschke, O. Shapoval, A. Belenchuk, J. Faupel, O. I. Lebedev, J. Verbeeck, G. Van Tendeloo, M. Mucksch, V. Tsurkan, R. Tidecks, and K. Samwer, Nat. Mat. 2, 247 (2003).

${ }^{34}$ O. I. Lebedev, J. Verbeeck, G. Van Tendeloo, O. Shapoval, A. Belenchuk, V. Moshnyaga, B. Damaschke, J. Faupel, and K. Samwer, Phys. Rev. B 66, 104421 (2002).

${ }^{35}$ V. Moshnyaga, I. Khoroshun, A. Sidorenko, P. Petrenko, A. Weidinger, M. Zeitler, B. Rauschenbach, R. Tidecks, and K. Samwer, Appl. Phys. Lett. 74, 2842 (1999).

${ }^{36}$ K. Gehrke, V. Moshnyaga, K. Samwer, O. I. Lebedev, J. Verbeeck, D. Kirilenko, and G. Van Tendeloo, Phys. Rev. B 82, 113101 (2010).

${ }^{37}$ M. Hÿtch, E. Snoeck, and R. Kilaas, Ultramicroscopy 74, 131 (1998).

${ }^{38}$ G. Catalan, Appl. Phys. Lett. 88, 102902 (2006).

${ }^{39}$ H. Tan, J. Verbeeck, A. Abakumov, and G. Van Tendeloo, Ultramicroscopy 116, 24 (2012). 
${ }^{40}$ D. A. Muller, L. F. Kourkoutis, M. Murfitt, J. H. Song, H. Y. Hwang, J. Silcox, N. Dellby, and O. L. Krivanek, Science 319, 1073 (2008).

${ }^{41}$ J. García-Barriocanal, F. Y. Bruno, A. Rivera-Calzada, Z. Sefrioui, N. M. Nemes, M. Garcia-Hernández, J. Rubio-Zuazo, G. R. Castro, M. Varela, S. J. Pennycook, C. Leon, and J. Santamaria, Adv. Mater. 22, 627 (2010).

${ }^{42}$ J. Simon, T. Walther, W. Mader, J. Klein, D. Reisinger, L. Alff, and R. Gross, Appl. Phys. Lett. 84, 3882 (2004).

${ }^{43}$ N. Nakagawa, H. Y. Hwang, and D. A. Muller, Nat. Mater. 5, 204 (2006).

${ }^{44}$ M. Varela, W. Grogger, D. Arias, Z. Sefrioui, C. León, C. Ballesteros, K. M. Krishnan, and J. Santamaria, Phys. Rev. Lett. 86, 5156 (2001).
${ }^{45}$ S. J. Pennycook, M. F. Chisholm, D. E. Jesson, D. P. Norton, D. H. Lowndes, R. Feenstra, H. R. Kerchner, and J. O. Thomson, Phys. Rev. Lett. 67, 765 (1991).

${ }^{46}$ J. Verbeeck, S. Bals, A. N. Kravtsova, D. Lamoen, M. Luysberg, M. Huijben, G. Rijnders, A. Brinkman, H. Hilgenkamp, D. H. A. Blank, and G. Van Tendeloo, Phys. Rev. B 81, 085113 (2010).

${ }^{47}$ S. A. Chambers, Surf. Sci. 605, 1133 (2011).

${ }^{48}$ H. Boschker, J. Verbeeck, R. Egoavil, S. Bals, G. Van Tendeloo, M. Huijben, E. P. Houwman, G. Koster, D. H. A. Blank, and G. Rijnders, Adv. Funct. Mater. 22, 2235 (2012).

${ }^{49}$ See Supplemental Material at http://link.aps.org/supplemental/ 10.1103/PhysRevB.87.035418 for a HRTEM image of a fully oxidized LMO/BTO superlattice on $\mathrm{MgO}(100)$. 